\title{
Self-adaption Image Enhancement Algorithm Based on Rule Fuzzy Inference Mechanism
}

\author{
Shenshutao ${ }^{1}$ and Gaofei ${ }^{2}$ \\ 1. Department of Tibetan information technology research center, Tibet \\ University, \\ Lhasa Tibet, 850000, China \\ 2. Department of scientific research University of Tibet, Tibetan university, \\ Tibet Lhasa, 850000 \\ 46210587@qq.com
}

\begin{abstract}
In order to solve such problems as background noise amplification, excessive image edge enhancement and ring effect in the output image caused by the difficulty of the existing self-adaption image enhancement algorithm in-identifying the smooth and fine areas of an image, a self-adaption image enhancement algorithm based on rule fuzzy inference system is proposed in this paper. Firstly, the pixel field of the image to be enhanced is locally and statically analyzed to obtain the low-frequency component of the image; secondly, five logic rules are defined and mednumile the local statistical information is combined with the local standard deviation to establish the rule fuzzy inference system so as to calculate the contrast gain actor and accordingly complete the self-adaption enhancement of the image and optimize the pixel field dimension. The simulation result shows: compared with existing contrast enhancement technology, the proposed technology has better visual enhancegment effect and can obviously eliminate ring effect.
\end{abstract}

Keywords: Image enhancement, Statistical analysis; Logic rule; Fuzzy inference; Local standard deviation

\section{Introdaction}

During image collection, various factors including dissatisfactory illumination intensity, poor camera hardware performance and manual operation can cause the collected image 0 have dissatisfactory visual quality such as low contrast ratio and accordingly bring inconvenience to the subsequent image analysis[1-2]. In order to improve the visual effect of such damaged image and clearly recover the image, scholars of different countries have designed the contrast self-adaption enhancement technology, wherein the self-adaption contrast enhancement is a method used for highlighting the target of the damaged image through a certain technology and meanwhile restraining or eliminating the image information able to influence the visual quality according to the initial requirements of the user [3]. As a relatively popular method in present research, the self-adaption contrast enhancement aims at amplifying the high-frequency component of the image through the non-sharp masking technology so as to recover the image. Although the existing self-adaption contrast enhancement technology can improve visual quality, yet it is difficult to identify the smooth and fine areas of an image, thus amplifying the background noise, excessively enhancing the image edge areas, and causing the ring effect in the output image, as well as requesting the further improvement of the enhancement quality. In order to solve above problems, a self-adaption image enhancement algorithm based on rule fuzzy inference system is proposed in this paper. 
Firstly, the pixel field of the enhanced image is locally and statically analyzed to obtain the low-frequency component of the image; then, five logic rules are defined and meanwhile the local statistical information is combined with the local standard deviation to establish the rule fuzzy inference system so as to calculate the contrast gain factor and accordingly complete the self-adaption enhancement of the image; finally, the enhancement effect of the proposed algorithm is verified through Matlab platform.

\section{Analysis of Traditional Self-Adaption Contrast Enhancement Principle}

In order to adopt the self-adaption contrast enhancement technology to process image $f(x, y)$, wavelet transform [7] is adopted to decompose the image into highfrequency component $m(x, y)$ and low-frequency component $s(x, y)$ :

$$
W_{f}\left(a, b_{x}, b_{y}\right)=\int_{-\infty}^{\infty} \int_{-\infty}^{\infty} f(x, y) \phi(x, y) d x d y
$$

Where $b x$ and $b y$ respectively represent the translations at $x$-axis and $y$ axis; $\phi(x, y)$ represents two-dimensional basic wavelet. For each pixel in the Image, the average image intensity can be adopted to calculate the low-frequency component thereof $m(x, y)$ :

$$
m(x, y)=\frac{1}{(2 n+1)^{2}} \sum_{k=x-n=y-n}^{x+n} \sum_{l=y}^{y+n} f(k \pi)
$$

Where $f(k, l)$ represents the intensity value at $(k, l)$; according to Model (2), the lowfrequency component can be deducted from the original image to form high-frequency component $m(x, y)$ :

$$
s(x, y)=f(x, y)-\ln (x, y)
$$

Then, according to Models (2) and (3), the contrast gain can be embedded to calculate enhanced image $g(x, y)$ so as to complete the self-adaption enhancement:

$$
g(x, y)=m(x, y)+G(x, y), y)
$$

Where $G(x, y)$ represents the contrast gain at $(x, y)$; for the self-adaption image enhancement technology, gain function $G(x, y)$ is self-adaptive to spatial domain.

$$
\begin{gathered}
G(x, y)=\frac{A}{S D(x, y)} \\
L S D(x, y)=\frac{1}{(2 n+1)} \sqrt{\sum_{k=x-n}^{+n} \sum_{l=y-n}^{y+n}\lfloor f(k, l)-m(k, l)\rfloor^{2}}
\end{gathered}
$$

Where $A$ represents a constant and $L S D$ represents the local standard deviation.

According to Models (4) (6), the self-adaption contrast enhancement technology can make the high-frequency component area have relatively low gain value to weaken the capability thereof for ring effect elimination, but make the low-frequency component area have relatively high gain value to easily amplify the background noise thereof, thus causing the output image to have poor visual quality. In the following example, the selfadaption enhancement technology is adopted to process Figure 1(a), and the result is as show $n$ in Figure 1(b). According to the two Figures, the enhancement quality is general and the enhanced image has ring effect as shown in Figure 1(b). Additionally, according to the histogram distribution thereof, compared with the original image (according to Figure 1(c)), the image processed by traditional self-adaption contrast enhancement technology has significant disadvantage: the distribution thereof is improved more or less, but such distribution is still uneven and part of the dynamic scope is lost, as shown in Figure 1(d)). 


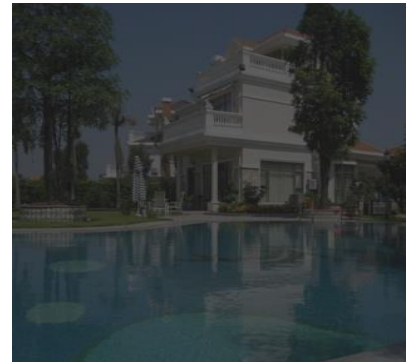

(a) Low-contrast Image

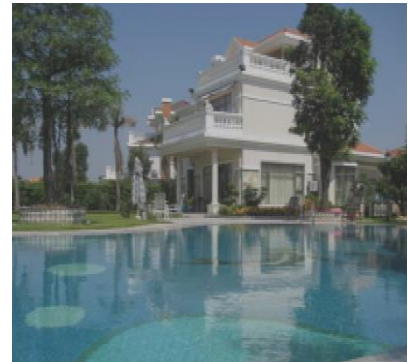

(b) Self-adaptively Enhanced Image

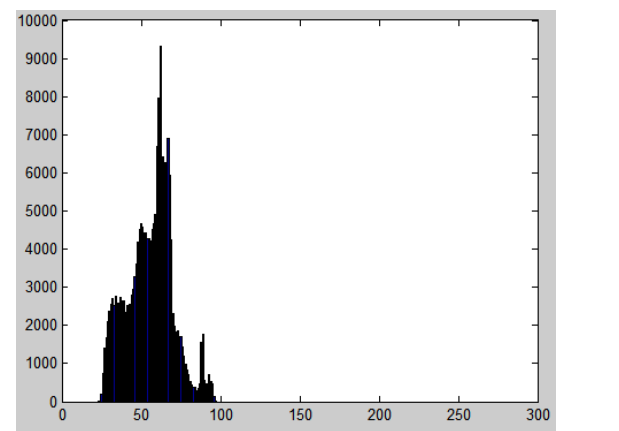

(c) Histogram Distribution of Low-contrast Image (Matlab Test)

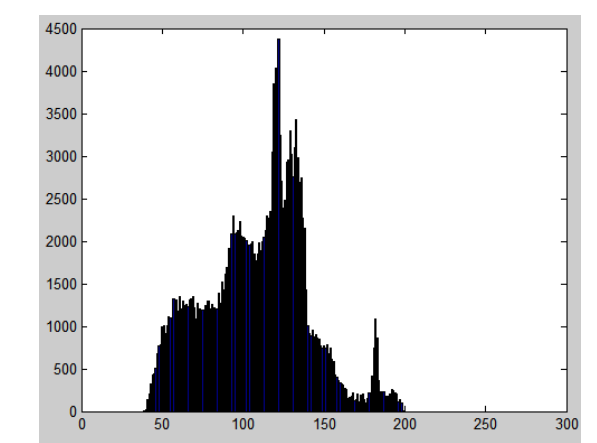

(d) Histogram Distribution of Self-adaptively Enhanced Image (Matlab Test)

\section{Figure 1. Classic Self-Adaption Image Enhancement Effect}

\section{Fuzzy Self-Adaption Contrast Enhancement}

As mentioned above, a suitable contrast gain value can significantly influence selfadaption effect. Therefore, a self-adaption image enhancement algorithm based on rule fuzzy inference system is proposed in this paper, as shown in Figure 2. According to Figure 2, this enhancement technology aims at collecting the local statistical characteristics of the image pixel field and then combining the local standard deviation to establish the fuzzy inference system so as to calculate the contrast gain value. Subsequently, according to enhancement gain value $G(x, y)$ obtained thereby, Model (4) is adopted to output the enhanced image. The parameters needed by the rule fuzzy inference system are extracted from the image to be enhanced, target image $f(x, y)$ and the field dimension are the input parameters of the system, and the output result is the enhanced image. Due to the fuzzy inference system adopted in the proposed algorithm, a suitable maximum gain value can be automatically endowed thereto for reducing excessive enhancement and ring effect. Obviously, the core part of the algorithm is the establishment of the rule fuzzy inference system. 


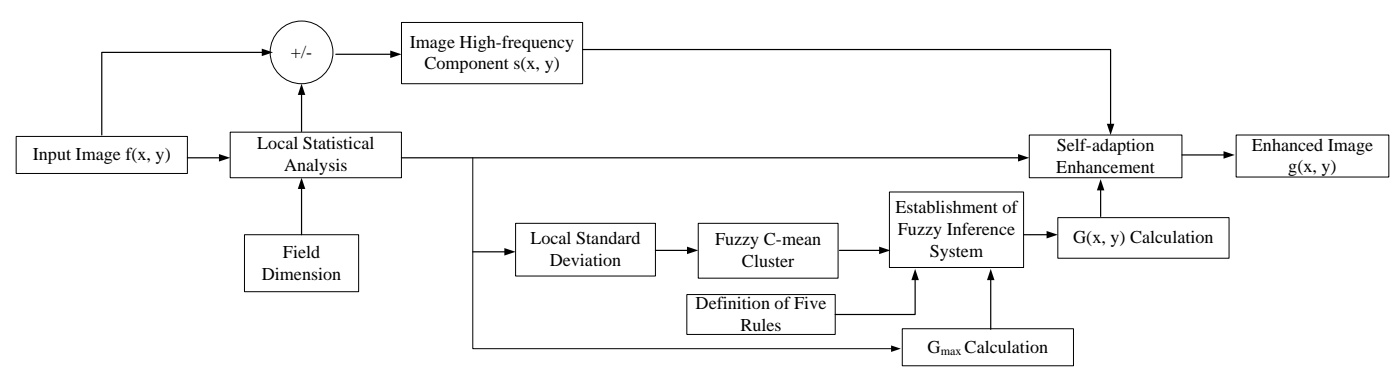

Figure 2. Algorithm Flow

\subsection{Establishment of Fuzzy Inference System}

Three conditions should be provided for designing the fuzzy inference system: (1) Define the parameter, shape and quantity of the fuzzy set to represent the input and output: field thereof; (2) Define fuzzy rules to add different combinations of the input and output fuzzy sets; (3) Adopt rational inference mechanism to determine the conclusion in the fuzzy rules defined thereby.

\subsubsection{Designation of Input and Output Fuzzy Sets}

The input field of the fuzzy self-adaption enhancementechnology is based on variable LSD (Local Standard Deviation) [8], wherein the local standard deviation is usually used to measure the local signal of the pixel field. Therefore, the input field is divided into five fuzzy sets in this paper: ultralow value, low value, median value, high value and ultrahigh value. Meanwhile, five membership function are adopted to represent five fuzzy sets: $\mu V L$ (LSD), $\mu L(L S D), \mu M(L S D), \mu h(C S D)$ and $\mu V h(L S D)$. Input $L S D$ values of the five fuzzy sets can be determined through these membership functions, and five fuzzy sets are adopted to replace three sets (low value, median value and high value) so as to provide smooth transition among the contrast gains of these fuzzy sets. Subsequently, it is necessary to designate the type and the parameter of the fuzzy sets. For the ultralow-value fuzzy set, $\mathrm{Z}$ membership fanction $\mu V L(L S D)$ is adopted in this paper, as shown in Figure 3(a). Specifically, the nodel thereof is as follows:

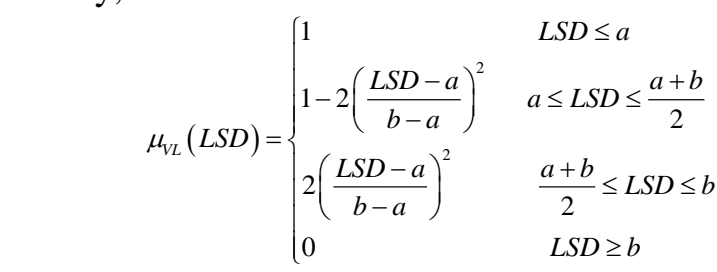

Where $a$ and $b$ respectively represent the extreme values of the curve slope.

According to Model (7) and Figure 3(a), if $L S C \leq a$ is true, then the membership degree grade thereoris 1 ; when $L S D$ is increased, the the probability for the input value to belong to the ultralow fuzzy set is reduced and the membership degree grade thereof starts to reduce. For low-value fuzzy set, median-value fuzzy set and high-value fuzzy set, Gaussian membership function is adopted in this paper, as shown in Figure 2(b), and the model thereof is as follows:

$$
\mu_{L, M, h}(L S D)=e^{-\frac{(L S D-c)^{2}}{2 d}}
$$

Where, $c$ and $d$ respectively represent the mean value and the variance of Gaussian function.

For the ultrahigh-value fuzzy set, S membership function is adopted in this paper, as shown in Figure 3(c), and the model thereof is as follows: 


$$
\mu_{V h}(L S D)=\left\{\begin{array}{lc}
0 & L S D \leq e \\
\left(\frac{L S D-e}{f-e}\right) & e \leq L S D \leq \frac{e+f}{2} \\
1-2\left(\frac{L S D-e}{f-e}\right)^{2} & \frac{f+e}{2} \leq L S D \leq f \\
1 & L S D \geq f
\end{array}\right.
$$

Where, $e$ and $f$ respectively represent the extreme values of the curve slope.

According to Model (9), for ultrahigh-value $L S D$, the probability for these values to belong to this fuzzy set is approximate to 1. Meanwhile, in order to define the input fuzzy sets, it is necessary to designate their parameters. Due to the excessive time consumption for parameter determination and the serious dependence on image contents, fuzzy C-mean cluster algorithm [9] is introduced in this paper to determine the parameters of these membership functions. Specifically, this algorithm is adopted to divide $\mathrm{N}$ data points containing $\mathrm{V}$ characteristics into $\mathrm{M}$ fuzzy groups and meanwhile find cluster center $\mathrm{Ci}$ of each group in order to minimize the differential measurement of the clusters. In the proposed algorithm, the input object of the fuzzy C-mean cluster algoritnm is $K \times 1$ dimensional eigenvector, including $L S D$ of $(2 n+1) \times(2 n+1)$ field centers around each pixel in the image, wherein $K$ is the total quantity of the image pixels; the input of the fuzzy Cmean cluster algorithm is the set of cluster centers $C i$ where n $\mathrm{C}=\{C V L, C L, C M, C h$, $C V h$. The corresponding five fuzzy sets are respectively $U V L, U L, U M, U h$, and $U V h$, including $L S D$ quantity of each cluster. Moreover the center and the variance of each cluster are adopted to define the parameter of the corresponding fuzzy set in the input field, as shown in Table 1.

Table 1. Type and Parameter of Membership Function of Input Fuzzy Set

\begin{tabular}{ccc}
\hline Fuzzy Set & Membersbip Function & Parameter \\
\hline Ultralow Value (VL) & Z Membership Function & $\mathrm{a}=\mathrm{CVL}, \mathrm{b}=\mathrm{CL}$ \\
Low Value (L) & Gaussian Membership Function & Variance of $\mathrm{c}=\mathrm{CL}$ and d=UL \\
Median Value (M0 & Gaussian Membership Function & Variance of $\mathrm{c}=\mathrm{CM}$ and d=UM \\
High Value (h) & Gaussian Membership Function & Variance of $\mathrm{c}=\mathrm{Ch}$ and d=Uh \\
Ultrahigh Value (Vh) & S Membership Function & Variance of e=Ch and $\mathrm{f}=\mathrm{UVh}$ \\
\hline
\end{tabular}

The output field of the (uzzy inference system proposed in this paper is gain function $G$, respectively divided into four fuzzy sets, namely: Gain1, Gain2, Gain3 and Gain4. Meanwhile, four fuz 2 single-row membership functions are adopted for representation: $\mu \operatorname{Gain} 1(G), \mu \operatorname{Gain} 2($ G),$\mu \operatorname{Gain} 3(G)$ and $\mu \operatorname{Gain} 4(G)$, thus indicating that the fuzzy inference system of the proposed technology in this paper is 0-order Sugeno system. Since Sugeno fuzzy inference system has relatively low complexity during the designation of the output fuzzy set, thus such system is superior to existing Mamdani and Tsukamoto systems. The fuzzy single-row membership function is as shown in Figure 3(d), and the member hip degree of the output fuzzy set is defined as follows:

$$
\mu G_{\text {ainz }}(G)=\left\{\begin{array}{ll}
1 & G=G_{z} \\
0 & \text { otherwise }
\end{array} \quad z=1,2,3,4\right.
$$

Where $G z$ represents a single data point.

For a rational maximum contrast gain Gmax, the fuzzy single-row position of the output field is as follows:

$$
\left\{\begin{array}{l}
G_{1}=1 \\
G_{2}=\frac{G_{3}+1}{2} \\
G_{3}=\frac{G_{\max }+1}{2} \\
G_{4}=G_{\max }
\end{array}\right.
$$




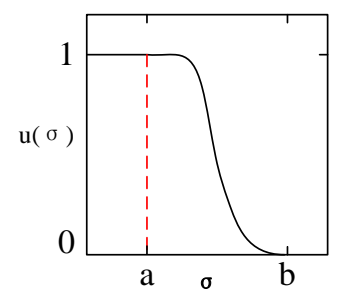

(a) Z Shape

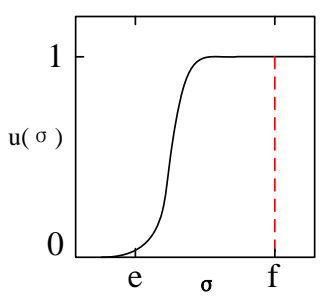

(c) S Shape

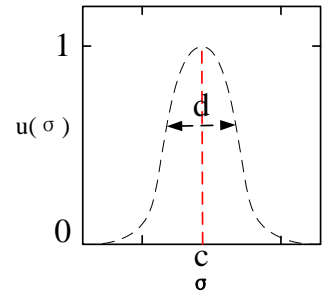

(b) Gaussian

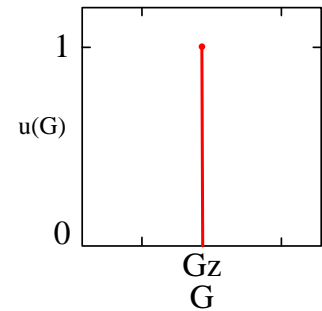

(b) Fuzzy Single-row

Figure 3. Type of Fuzzy Sets Used For Designing Rule Fuzzy Inference System of the Proposed Algorithm

\subsubsection{Definition of Rules of Fuzzy Inference System}

After defining the input and output fuzzy sets, it is necessary to design the rules of the inference system. Based on $L S D$, the following five rules ate defined in this paper:

(a) Rule 1: if $\operatorname{LSD}(x, y)$ is an ultralow value, then $G(x, y)$ is Gain1;

(b) Rule 2: if $\operatorname{LSD}(x, y)$ is a low value, then $G(x, y)$ is Gain2;

(c) Rule 3: if $L S D(x, y)$ is a median value, then $G(x, y)$ is Gain4;

(d) Rule 4: if $\operatorname{LSD}(x, y)$ is a higlvalue, then $G(x, y)$ is $\operatorname{Gain} 3$;

(e) Rule 5: if $\operatorname{LSD}(x, y)$ is an ultrahigh value, then $G(x, y)$ is $\operatorname{Gain1}$;

According to the above five rules, for the self-adaption enhancement technology, Rule 1 and Rule 5 are related the low-signal and strong-signal image areas and the low gain value is endowed thereto. Therefore, for an ultralow or ultrahigh $L S D$ value, the gains of all rules are approximate to 1 , thus indicating that the change of the corresponding image pixel is minimized and such minimized change is very important to reduce noise amplification and ring effect. Rule 3 is related to the median value of the image and the maximum gain value is endowed thereto, thus to maximally change pixel intensity. $L S D$ values of Rule 2 and Rule 4 are corresponding to the transition from the smooth area of the image to the intermediate detail area of the image and the transition from the intermediate detan area to the rich detail area, and the suitable gain value is endowed thereto. Since transition usually appears in the smooth area and the intermediate detail area, thus the contrast gain value of Rule 2 is lower than that of Rule 4. Therefore, for all $L S D$ values, the gain values endowed thereto are different from each other. Oppositely, these gain values are fuzzy, and the gain value of the cluster quantity is determined acco ding to the membership degree.

\subsubsection{Fuzzy Inference Mechanism}

Finally, the inference mechanism is designed on the basis of the inference combination rules. In order to determine the output value according to the preset input value, the contrast gain value of the image pixel is obtained on the basis of $L S D$ value in the fuzzy self-adaption enhancement technology. Namely: the inference mechanism is adopted to add $L S D$ value to the pixel located at $(x, y)$ in $L S D$ membership function $\mu g(k)$ of the input fuzzy set. Specifically, the calculation model for contrast gain $G(x, y)$ thereof is as follows: 


$$
G(x, y)=\frac{\sum_{k=1}^{5} \mu_{Q(k)}(L S D(x, y)) G_{I(k)}}{\sum_{k=1}^{5} \mu_{Q(k)}(L S D(x, y))}
$$

Where $k$ represents the quantity of the rules, $Q=(\mathrm{V} L, L, M, h, V h)$ represents the type of the input fuzzy set, and $\mathrm{I}=(1,2,3,4,1)$ represents the subscript of the output gain set.

Obviously, Model (12) is the mean value of $L S D$ membership degree grade in each weighted fuzzy set and means that the contrast gain value not only depends on the affiliation degree of $L S D$ to a certain fuzzy set, but also depends on the membership degrees of other fuzzy sets. Therefore, the gain value is mainly determined by $L S D$ at ( $x$, $y$ ) and is related to the local signal of the whole image.

The enhancement result of Figure 1(a) processed by the proposed fuzzy inference mechanism is as shown in Figure 4(a). Obviously, the image processed thereby has good visual effect, uniform histogram distribution and relatively complete dynamic scope, without obvious ring effect, as shown in Figure 1(b).

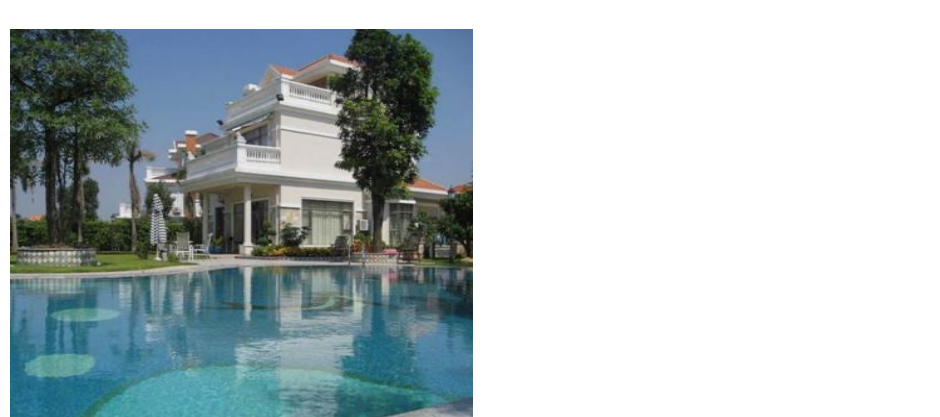

(a) Image enhanced by the proposed fuzzy inference system

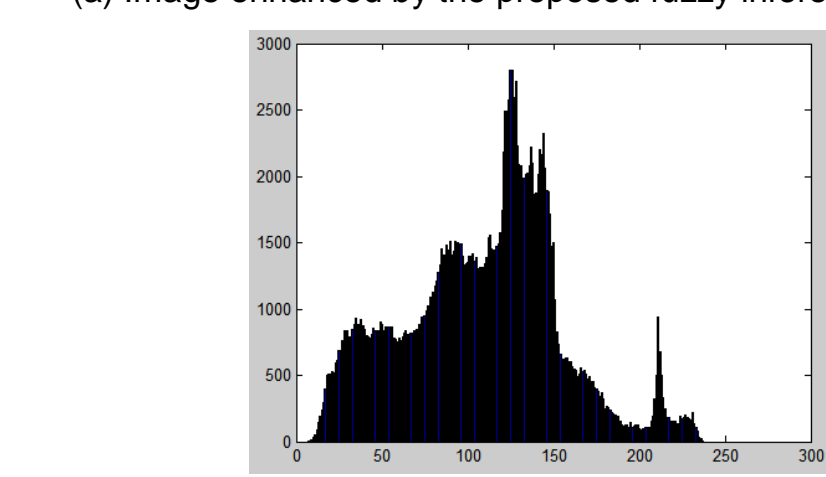

(b) Histogram distijbution of image output by the proposed fuzzy inference system (matlab test)

Figure 4. Enhancement Quality of the Proposed Algorithm and Histogram Distribution

\section{Simalation Test and Analysis}

In order to reflect the enhancement effect of the proposed algorithm, the enhancement performance and the visual quality are tested through Matlab tools. Meanwhile, the existing algorithms with relatively good self-adaption enhancement effect are taken as the control group: literatures [4] and [10] are respectively regarded as Algorithms A and B, wherein average edge gray value $\bar{E}$ and reference image definition measurement TEN [11] are introduced therein to quantify the edge intensity and the visual quality of the enhancement image:

$$
\bar{E}=\frac{1}{M N} \sum_{x=1}^{M} \sum_{y=1}^{N} Q(x, y)
$$




$$
\begin{gathered}
Q(x, y)=\frac{\sum_{k=x-n}^{x+n} \sum_{l=y-n}^{y+n} \Delta(k, l) f(k, l)}{\sum_{k=x-n}^{x+n} \sum_{l=y-n}^{y+n} \Delta(k, l)} \\
T E N=\sum_{x} \sum_{y} \nabla(x, y) \quad \forall G(x, y)>\lambda
\end{gathered}
$$

Where $(x, y)$ represents image pixel, $\Delta(k, l)$ represents the edge value obtained and calculated on the basis of the edge operator, $M N$ represents the image dimension, $\nabla(x, y)$ represents the gradient value, $\lambda$ represents the threshold value for edge effect elimination, and $\lambda$ is set as the mean value of the gradient in this paper.

\subsection{Determination of Pixel Field Dimension Optimization}

In the proposed fuzzy self-adaption enhancement technology, the key parameter is the maximal rational contrast gain value $\operatorname{Gmax}(x, y)$, as shown in Model (13), and this value is significantly influenced by field $W x y$ with the dimension as $(2 n+1) \mathrm{x}(2 n+1)$. The larger pixel field can generate wider intensity scope. In other words, lower value is endowed to $W_{x y}^{\min }$ while higher value is endowed to ${ }^{W_{x y}^{\max }}$, thus to finally form greater gain alue $\operatorname{Gmax}(x$, $y)$. However, larger $\operatorname{Gmax}(x, y)$ can weaken the capability for ring effect elimination. Therefore, pixel field dimension $n$ can significantly influence the enhancement performance of the proposed algorithm, and $n$ valae should be optimized before experimental test. Specifically, Figure 1(a) is taken as an example to opimally determine $n$ value. After the image is processed by the proposed algorithm, the test results of Gmax, average edge gray value, reference image definition and PSNR (Peak Signal to Noise Ratio) are as shown in Figures 5(b) (e) According to the figures, along with the increase of $n$ value, Gmax, edge gray value and reference image definition TEN are firstly increased and then reduced, but PSNR value is reduced. Therefore, the combined weight $T=G \max +E+T E N+P S N R$ is defined in this paper to determine suitable $n$ value. According to Figure $5, n$ is finally determine $2 \mathrm{~s} n=3$.
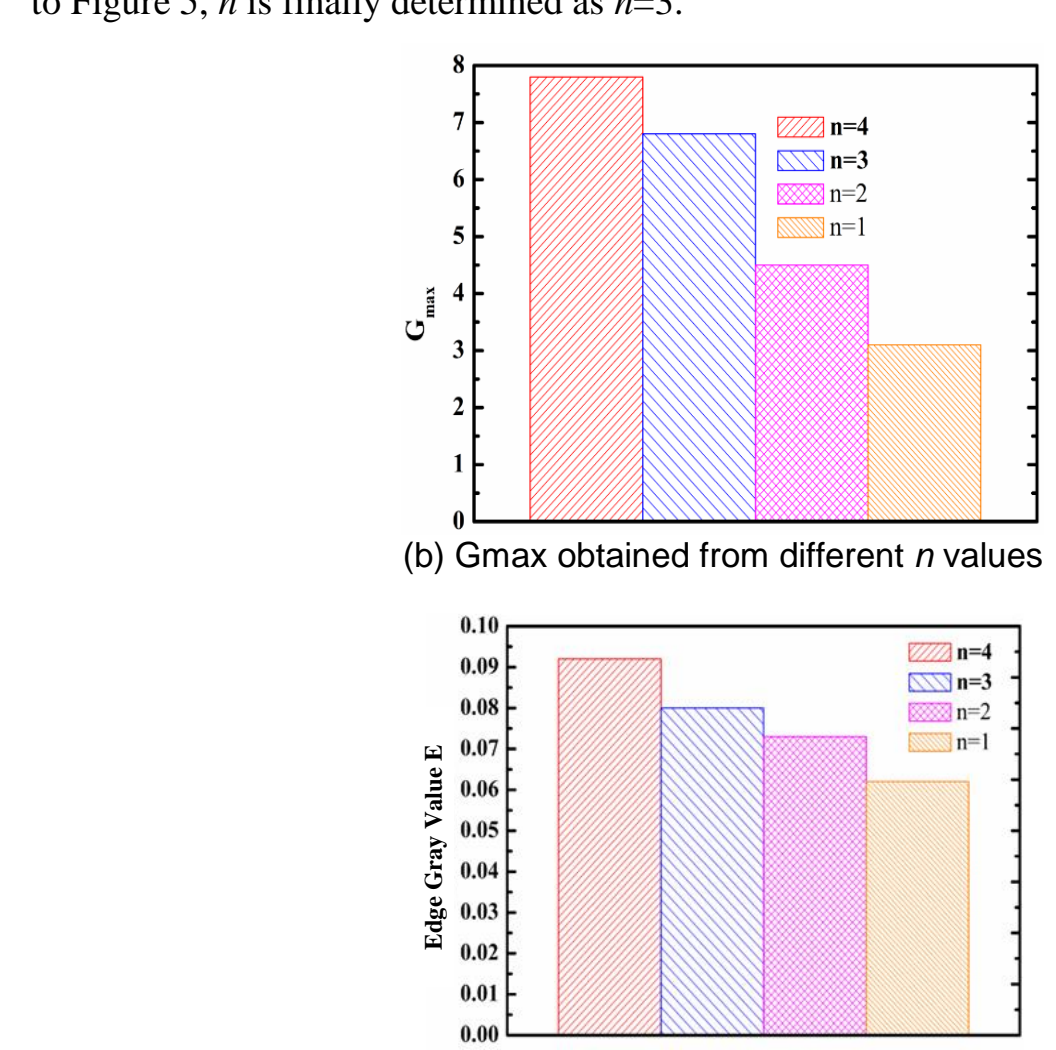

(c) E value obtained from different $n$ values 

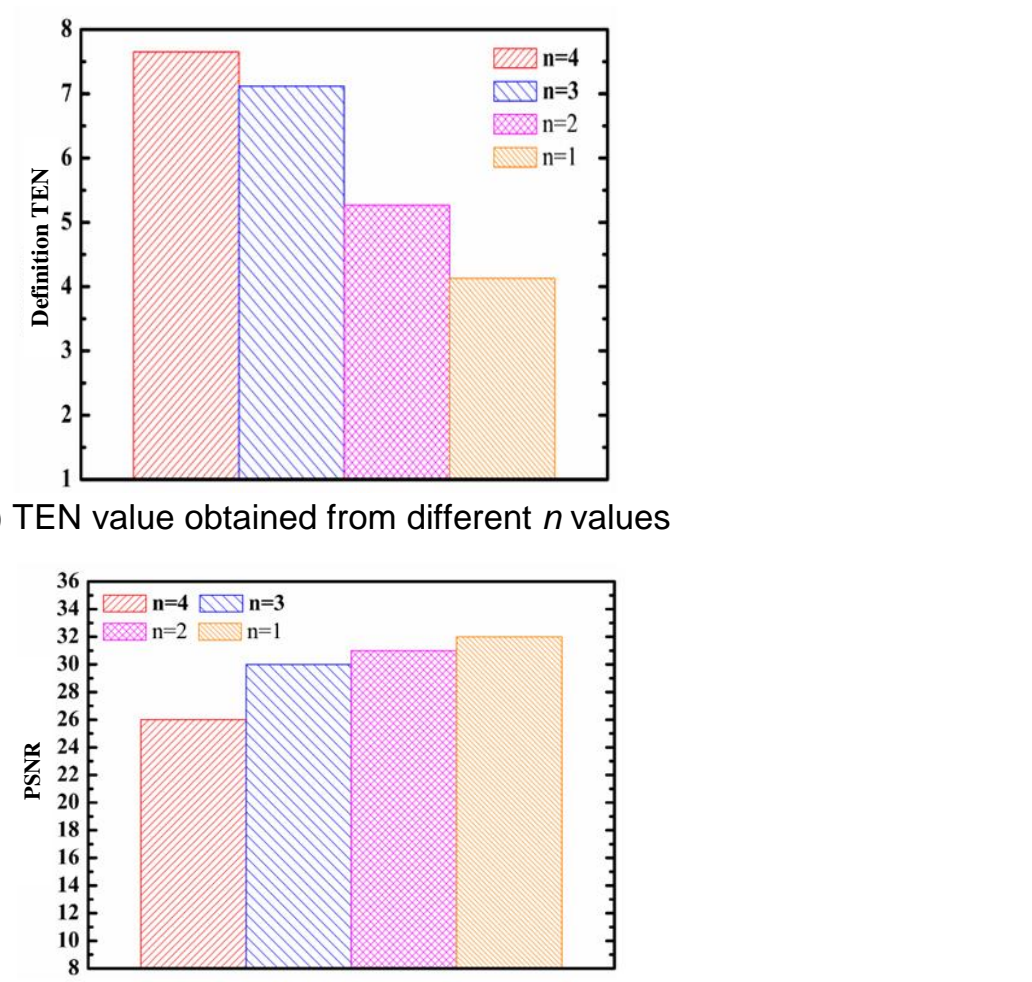

(e) PSNR value obtained different $n$ values

Figure 5.Test Results of Quantitative Indexes Under Different Pixel Field Dimensions

\subsection{Enhancement Effect Analysis}

The low-contrast image captured by Canon camera (EOS450+1/1.8' CCD sensor) is taken as the test sample, as shown in Figure 6(a). The quality enhancement effects of the proposed algorithm and Algorithms $\mathrm{A}$ and B are as shown in Figure (b) (d). According to the figures, the image enhanced by the proposed algorithm has the best visual effect and the lowest distortion anc can significantly reduce noise amplification and ring effect, without image color change or excessive enhancement, as shown in Figure 6(b). However, the algorithms in the control group have ideal visual effect of the contrast enhancement, distorted output result and ring effect in the recovered image, and are difficult to reduce noise amplification, as shown in Figures 6(c) (d). The reason for the above result is as follows: the fuzzy inference system is adopted in the proposed algorithm to endow the suitable maximum contrast gain values to different areas so as to realize mooth transition and dynamically adapt to the intensity scope, but the algorithms in the control group aim at overall completing the enhancement rather than considering the 1ow-frequency and high-frequency component areas of the image, thus to be difficult to eliminate the ring effect of the image edge area. 


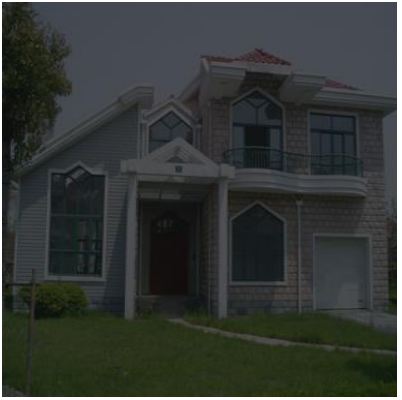

(a) Low-contrast sample

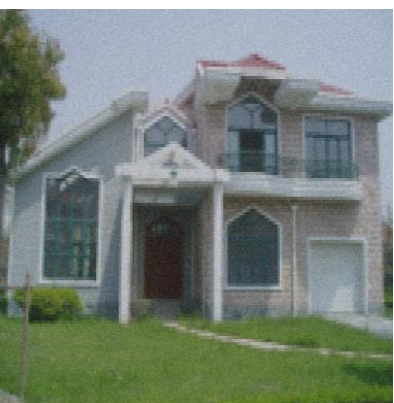

(c) Visual enhancement of the algorithm in literature [4]

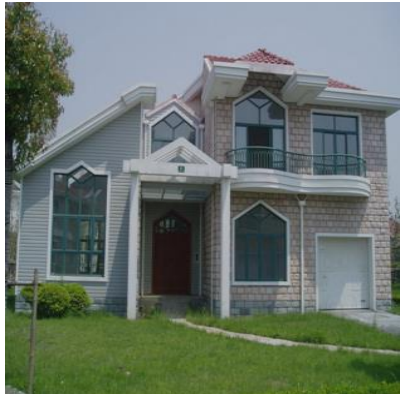

(b) Visual enhancement of the proposed algorithm

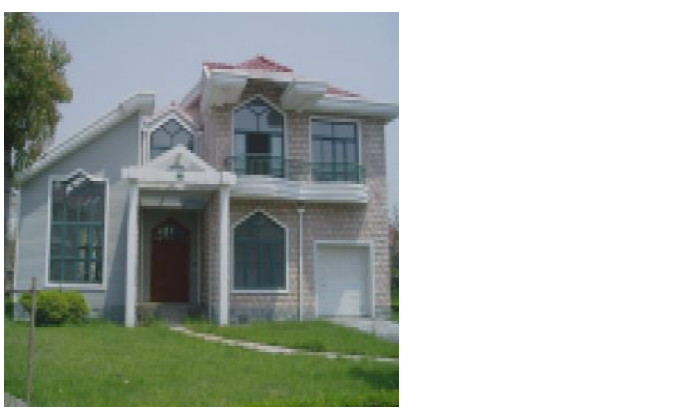

(d) Visual enhancement

of the algocithm in literature [10]

Figure 6. Visual Enhancement Effects of Different Self-Adaption Algorithms

\section{Conclusion}

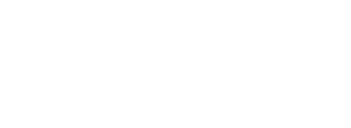

In order to eliminate the excessive enhancement and ring effect in existing contrast enhancement technology a self-adaption image enhancement algorithm based on rule fuzzy inference system is proposed in this paper. Firstly, the pixel field of the image to be enhanced is locally and staticaly analyzed to obtain the low-frequency component of the image; secondly, five logic rules are defined and meanwhile the local statistical information is combined with the local standard deviation to establish the rule fuzzy inference system so as to calculate the contrast gain factor, and the maximum rational contrast gain calculation model is also established on the basis of the high-frequency component $s(x, d)$ of the image so as to complete the self-adaption enhancement of the image. The test result shows: compared with existing contrast enhancement algorithms, the proposed technology has better visual enhancement effect, larger average edge gray value and definition, and can obviously reduce noise amplification and eliminate ring effect.

\section{Acknowledgements}

The research is supported by Key projects of science and Technology Department of Tibet autonomous region (Z2013B28G28/02). 


\section{References}

[1] D. Jiang, X. Ying and Y. Han, "Collaborative multi-hop routing in cognitive wireless networks", Wireless Personal Communications, vol. 86, no.2, (2016), pp. 901-923.

[2] Z. Lv, A. Halawani and S. Feng, "Multimodal hand and foot gesture interaction for handheld devices", ACM Transactions on Multimedia Computing, Communications, and Applications (TOMM), vol. 11, no. $1 \mathrm{~s}$, (2014).

[3] J. Hu and Z. Gao, "Distinction immune genes of hepatitis-induced heptatocellular carcinoma", Bioinformatics, vol. 28, no. 24, (2012), pp. 3191-3194.

[4] J. Hu, Z. Gao and W. Pan, "Multiangle Social Network Recommendation Algorithms and Similarity Network Evaluation", Journal of Applied Mathematics, vol. 2013, (2013).

[5] J. Hu and Z. Gao, "Modules identification in gene positive networks of hepatocellular carcinoma using Pearson agglomerative method and Pearson cohesion coupling modularity", Journal of Applied Mathematics, (2012).

[6] Y.Geng, J.Chen, R. Fu, G. Bao and K. Pahlavan, "Enlighten Wearable Physiological Monitoring systems: On-Body RF Characteristics Based Human Motion Classification Using a Support Vector Machine", vol. PP, no. 99, pp. 1-16.

[7] X. Song and Y. Geng, "Distributed Community Detection Optimization Algorithm for Complex Networks", Journal of Networks, vol. 9, no. 10, pp. 2758-2765.

[8] K. Pahlavan, P. Krishnamurthy and Y. Geng, "Localization Challenges for the Emergence of the Smart World", Access, IEEE, vol. 3, no. 1, pp. 1-11.

[9] J. He, Y. Geng, Y. Wan, S. Li and K. Pahlavan, "A cyber physical test-bed for yirtualization of RF access environment for body sensor network", Sensors Journal IEEE, vpl. 13, no. 10, 2013), pp. 38263836.

[10] D. Jiang, Z. Xu and Z. Lv, "A multicast delivery ap roach with minimum energy consumption for wireless multi-hop networks", Telecommunication Systems, (2015), pp. 112

[11] Y. Lin, J. Yang and Z. Lv, "A self-assessment stereo capture model applicable to the internet of things",Sensors, vol. 15, no.8, (2015), pp. 20925-20944.

[12] Y. Liang*, "Satisfaction with Economic and So cial Rights and Quality of Life in a Post-Disaster Zone in China: Evidence From Earthquake-Prone Sichuan", Disaster Mledicine and Public Health Preparedness, vol. 9, no. 2, pp. 111-118.

[13] Y. Liang*, "Correlations Between Health-Related Quality ofLife and Interpersonal Trust: Comparisons Between Two".

[14] "Generations of Chinese Rural-to-Urban Migrant_Social Indicators Research", vol. 123, no. 3, pp. 677700.

[15] Y. Liang* and P. Lu, "Medical insurance policy organized by Chinese government and the health inequity of the elderly: longitudinal comparison based on effect of New Cooperative Medical Scheme on health of rural elderly in 22 provinces and cities", International Journal for Equity in Health, vol. 13, no. 37, (2014), pp. 115.DQI:10.1186/1475-9276-13-37.

[16] Y. Liang* and D Zhu, "Subjectve Wel-Being of Chinese Landless Peasants in Relatively Developed Regions: Measurement Using PANAS and SWLS. Social Indicators Research”, vol. 123, pp. 3, 817-835.

[17] Y. Liang* and X. Wang, Deyeloping a new perspective to study the health of survivors of Sichuan earthquakes in China: astudy on the effect of post-earthquake rescue policies on survivors' healthrelated quality of life", Health Research Policy and Systems, vol. 11, pp. 41, 1-12. DOI:10.1186/14784505-11-41.

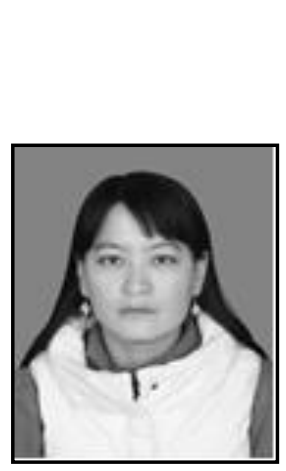

Authors

Shen Shutao, She received her M.S. degree in Computer Application Major from Southwest Jiao Tong University in Sichuan, China. She is a lecturer at the University of Tibet Tibetan Information Technology Research Center Her research interests is mainly in the area of computer image processing, She has published several research papers in scholarly journals in the above research areas And several projects have been applied. 
International Journal of Hybrid Information Technology

Vol. 9, No.9 (2016)

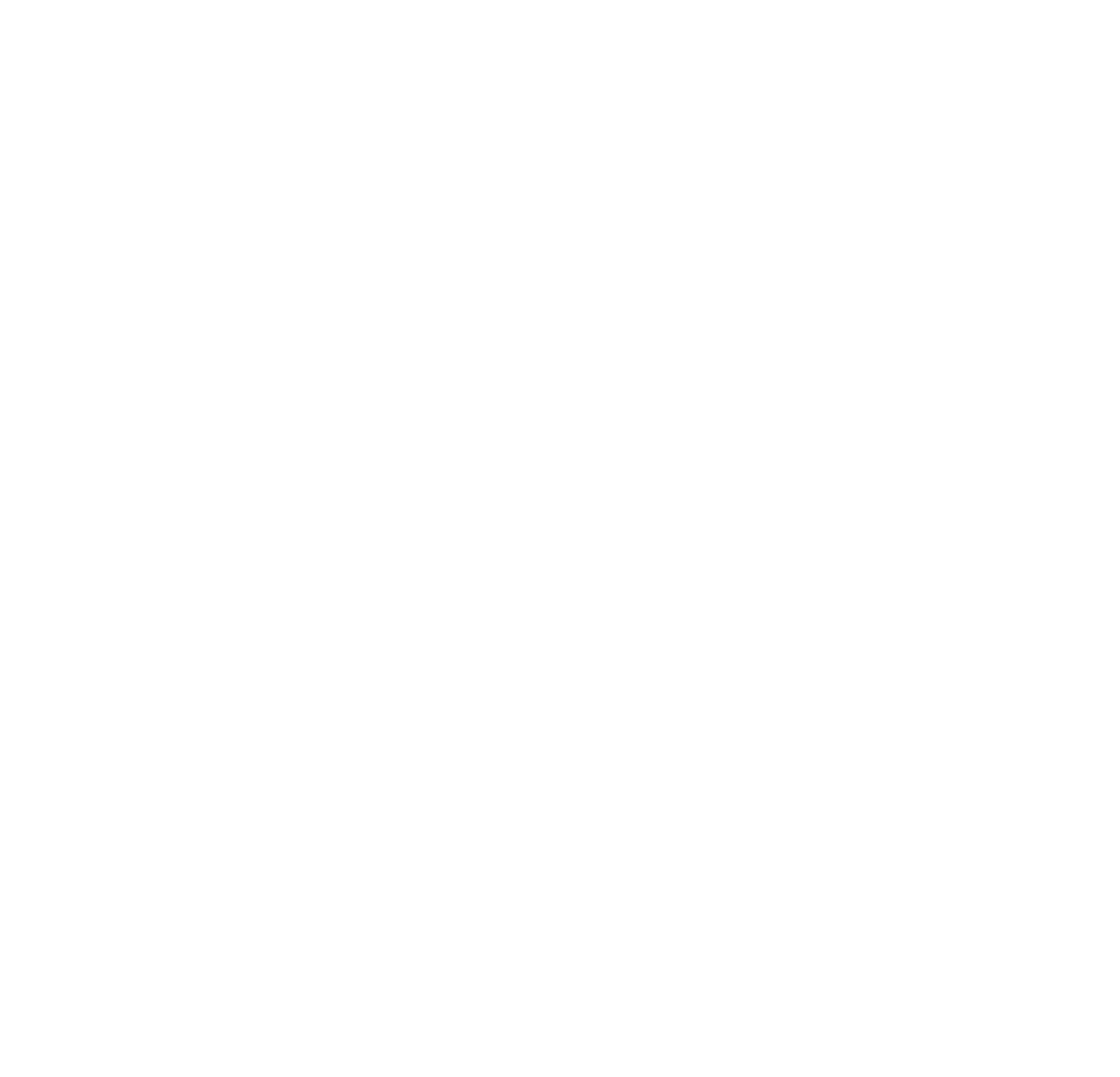

